\title{
Microbiological and antibiotic sensitivity pattern of surgical site infection following caesarean section in a tertiary care center of Chhattisgarh
}

\author{
Neha Thakur ${ }^{1 *}$, Avinashi Kujur ${ }^{2}$ \\ ${ }^{1}$ Department of Obstetrics and Gynecology, Pt. JNM Medical college, Raipur, Chhattisgarh, India \\ ${ }^{2}$ Department of Obstetrics and Gynecology, Govt. Medical College Ambikapur, Chhattisgarh, India
}

Received: 25 May 2021

Accepted: 14 June 2021

*Correspondence:

Dr. Neha Thakur,

E-mail: neha051988@gmail.com

Copyright: $\odot$ the author(s), publisher and licensee Medip Academy. This is an open-access article distributed under the terms of the Creative Commons Attribution Non-Commercial License, which permits unrestricted non-commercial use, distribution, and reproduction in any medium, provided the original work is properly cited.

\section{ABSTRACT}

Background: It is one of the most common surgery practiced in present era in obstetric population it has led to rise in postoperative morbidity in the form of surgical site infection (SSI). It not only burdens the health system but it also puts a serious negative impact on patient's life. The present study is aimed to determine the incidence, risk factors and the microbiological and antibiotic sensitivity pattern for SSI following caesarean section in our institute.

Methods: The study is prospective, descriptive study carried out in department of obstetrics and gynecology of Pt. Jawaharlal Nehru medical college, Raipur between May 2019 and April 2020.

Results: Out of the 1215 participants who underwent caesarean section, 251 patients had SSI, with incidence of 20.6\% among them superficial SSI $(n=154)$ was $61.3 \%$ while deep SSI $(n=75) 29.8 \%$ and $8.7 \%(n=22)$ organ/space SSI. Gram positive organism was highest isolated from superficial SSI $66.2 \%$ while from deep SSI and organ/space SSI gram positive organism isolated was $45.2 \%$ and $54.2 \%$ respectively, gram negative organisms were maximum isolated from organ/space SSI 40.9\% followed by deep and superficial SSI 36\% and 34.4\% respectively. Most isolates were highly resistant to cephalosporins, gentamycin and amoxicillin; moderately resistant to fluoroquinolones and highly sensitive to vancomycin, carbapenem and linezolid.

Conclusions: Our tertiary centre had post caesarean SSI rate of $20.6 \%$ which was high. By analyzing the microbiological and sensitivity pattern we can use evidenced-based sensitive antibiotics to be commenced initially when wound infection is identified in our wards while awaiting the result of wound swab microscopy, culture and sensitivity (48-72 hours), to individualize our antibiotic prophylaxis and postoperative antibiotic protocol policy to reduce the further complications.

Keywords: Caesarean section, Surgical site infection, Wound infections, Antibiotic sensitivity

\section{INTRODUCTION}

Post 1860's introduction of the antisepsis principles substantially decreased the post-operative infectious morbidity. Nevertheless, SSI remained as one of the major nosocomial infections amongst hospitalized patients. ${ }^{1}$ Since the first cesarean section reported, there has been tremendous improvement in surgical, anesthetic and peri and postoperative management with a resultant significant reduction in postoperative mortality as well as morbidity. Though, emerged as a safe mode of delivery, as shown by a rising trend in cesarean section rate in present era. ${ }^{2}$ It is one of the most common surgery practiced in present era in obstetric population. A rising trend in the caesarean section rate is observed all over the world in past 30 years. ${ }^{2}$ It has resulted in improvement in perinatal outcome but at the same time there is an increase in the postoperative morbidity particularly surgical site wound infection. 
According to CDC's national nosocomial infection surveillance (NNIS) system $38 \%$ of all nosocomial infections in surgical patients are SSI. They constitute third most common nosocomial infection. ${ }^{4}$

The rates of SSI after caesarean deliveries range from the incidence varies from $6 \%$ to $27 \%$ depending on the type of operation, underlying patient status, associated co morbidities and surveillance methods used to identify infections, Studies in India have consistently shown higher rates ranging from $23-28 \% .^{3,5}$ In our study we observed incidence of SSI to be $20.5 \%$ (251 out of 1215) which is high, however, comparable to the incidence reported in our country. ${ }^{6}$

WHO defines SSI clinically as: "a purulent discharge around the wound or the insertion site of the drain or spreading cellulites from the wound." (CDC) National nosocomial infection surveillance (NNIS) system classifies SSIs as being either incisional or organ/space, occurring within 30 days after the operation. Incisional SSIs are further divided into those involving only skin and subcutaneous tissue (superficial incisional SSI) and those involving deeper soft tissues of the incision (deep incisional SSI). Organ/space SSIs involve any part of the anatomy (e.g., organ or space) other than incised body wall layers that was opened or manipulated during an operation. ${ }^{1}$

Surgical site wound infection is the most important associated morbidity with any kind of the surgery apart from the inherent risk of the surgery and the anesthesia. Knowledge regarding risk factors for SSI is mandatory; to develop prevention strategies and further reducing risk of infection.

The beneficial effect of antibiotic prophylaxis in reducing occurrences of infection associated with elective or emergency cesarean section is already well established. ${ }^{7,8}$ It also reduces the risk of endometritis and incisional SSI when administered correctly. $S$. aureus is the most commonly isolated bacteria in wound infections following cesarean section. ${ }^{9}$ This organism causes serious infections and has been shown to be resistant to commonly available, cheap antibiotics like the penicillin's. ${ }^{10}$ Other workers isolated more gram-negative organisms like E. coli, Proteus mirabilis, Pseudomonas and Klebsiella in CS wound infections. ${ }^{11}$ The variation in the spectrum of causative organisms means that prophylactic antibiotic though effective may fail when the wrong agent is used or used inappropriately, ${ }^{10}$ So, it is also important to know the sensitivity spectrum of the local microorganisms.

Therefore, conducting present study to know common organism and their sensitivity patterns for prompt management of patients and to commence sensitive antibiotic timely while waiting for wound swab microscopy culture and sensitivity in 48-72 hours, this will help in establishing our own department guideline for prevention and management of SSIs.

\section{METHODS}

The study is prospective, descriptive study carried out in department of obstetrics and gynecology of Pt. Jawaharlal Nehru medical college, Raipur between May 2019 and April 2020. A total of 1215 women undergone cesarean section in department were considered as eligible, for inclusion in the study.

Clearance from ethical committee was taken along with consent from all eligible patients to participate in the study. A total of 1215 women undergone cesarean section in department were considered as eligible, for inclusion in the study. Incidence demographic profile and risk factors were analyzed and Direct wound observation was done from postoperative day, all post caesarean SSI wounds were categorized as per CDC criteria into superficial, deep and organ/space SSI followed by wound swabs for microbiological culture were taken from the infection site using sterile swab stick before the wound is cleaned and sent to hospital microbiology department for culture sensitivity.

As per our hospital protocol we administer triple antibiotic in postoperative period having a combination of cephalosporin, aminoglycoside and metronidazole comprising of (1 gram ceftriaxone 12 hourly, gentamycin $80 \mathrm{mg} 12$ hourly and metronidazole $100 \mathrm{cc} 8$ hourly) for 5 days. This triple line antibiotic gives protection against gram positive cocci, gram negative bacilli and anaerobic organisms.

\section{Inclusion criteria}

Study population comprised of patients that had a cesarean section and then developed SSI during hospital stay.

\section{Exclusion criteria}

Patient who developed infection after discharge were not included in the study due to incompleteness of follow up and outside operated cesarean section and presenting in our hospital as SSI were excluded.

\section{RESULTS}

During the above-mentioned period a total of 2,800 no. of caesarean sections were done in the department. Out of them a total of 1215 cases, fulfilled the selection criteria. Out of these selected 1215 cases surgical site wound infection developed in 251 cases, giving an incidence of $20.6 \%$.

Majority of the patients with SSI were teenage (67.5\%), gravidity has shown a significant association with SSI $(\mathrm{p}<0.0001)$ with increasing gravidity risk for developing SSI increases by 3.3 folds; highest incidence in multigravida $(53.9 \%)$, rural population had maximum incidence $(26.5 \%)$, SES there is linear increase in risk for SSI with decreasing SES, highest among class V (74.3\%), 
in relation to gestational age incidence was highest in post term pregnancies $27.7 \%$. Among 89 cases with smoking/tobacco chewing 57 cases developed SSI with incidence of $64 \%$ maximum were smokers with incidence of $69.3 \%$ and $61.5 \%$ in tobacco chewers.

With increasing BMI there is 8-10 folds increase risk of SSI highest risk among morbidly obese $39.5 \%(\mathrm{p}<0.005)$. Grade of anemia has a direct relationship with SSI highest among severely anemic cases 30\% (35/117), p value of $=0.002$.

Ruptured membrane was an independent factor for developing SSI, statistically highly significant with RR $2.13(1.26-2.69 ; \mathrm{p}<0.00001)$ and incidence of $31.2 \%$, it was observed that $100 \%$ cases with membrane ruptured for $>24$ hours developed SSI with RR 6.4 (5.52-7.6; $\mathrm{p}<0.0001)$ showing linear relationship between development of SSI and duration of membrane rupture.

On univariate analysis of grade of surgeon as risk factor it showed independent association with SSI OR5.5 (95\% CI 3.3-9.3; $\mathrm{p}<0.0001)$.
On analyzing a ROC curve, we observed a cut off mean hemoglobin loss of $2.2 \mathrm{gm} / \mathrm{dl}$ indicating a sensitivity of $84.5 \%$ and specificity of $78.9 \%$ with OR 1.96 (1.23-6.59, $\mathrm{p}<0.0001)$. Cases having an intraoperative hemoglobin loss of $>2.2 \mathrm{gm} / \mathrm{dl}$ predisposes the patient to develop SSI. Cut off of $2.2 \mathrm{gm} / \mathrm{dl}$ indicates an estimated blood volume loss of $750 \mathrm{ml}$. In univariate analysis intraoperative blood loss has been observed as independent risk factor for SSI development.

Incidence of SSI was $24 \%$ among cases who received blood transfusion as against $18 \%$ of cases with no blood transfusion RR 1.33 (1.07-1.66, p=0.01) indicating significant relationship with SSI.

Mean duration of surgery for controls was $56.32 \pm 20.47$ whereas cases had mean $77.8 \pm 22.4$ on comparing there mean significantly higher duration of surgery was noted in case group $(\mathrm{p}<0.0001)$.

Vertical skin incision significantly increases the risk of SSI by 2 folds as among cases with transverse skin incision, RR $0.39(0.27-0.56, \mathrm{p} \leq 0.0001)$.

Table 1: Demographic profile of women with SSI following caesarean section.

\begin{tabular}{|c|c|c|c|c|c|}
\hline Variable & $\begin{array}{l}\text { Total no. of SSI } \\
\text { cases, }(n=251)\end{array}$ & Incidence & Mean \pm SD & OR $(95 \% \mathrm{CI})$ & P value \\
\hline \multicolumn{6}{|l|}{ Age (Years) } \\
\hline$<20(n=37)$ & 25 & 67.5 & \multirow{6}{*}{$25.05 \pm 4.33$} & & \multirow{6}{*}{-} \\
\hline $20-24.9(n=583)$ & 97 & 16.6 & & & \\
\hline $25-29.9(n=410)$ & 55 & 13.4 & & & \\
\hline $30-34.9(n=97)$ & 19 & 19.5 & & & \\
\hline $35-39.9(n=77)$ & 51 & 66.2 & & & \\
\hline$>40(n=11)$ & 4 & 36.3 & & & \\
\hline \multicolumn{6}{|l|}{ Gravidity } \\
\hline $1(n=497)$ & 100 & 20.1 & \multirow{3}{*}{$1.68 \pm 0.69$} & $1.52(1.25-1.81)^{*}$ & $<0.0001$ \\
\hline $2(n=588)$ & 81 & 13.7 & & & - \\
\hline$\geq 3(n=130)$ & 70 & 53.9 & & & - \\
\hline \multicolumn{6}{|l|}{ Gestational age } \\
\hline Preterm $(n=87)$ & 10 & 11.4 & \multirow{3}{*}{-} & - & - \\
\hline Term $(n=1020)$ & 211 & 20.6 & & $2.0(1.0-3.9)$ & 0.04 \\
\hline Post-term $(n=108)$ & 30 & 27.7 & & $2.9(1.35-6.4)$ & 0.006 \\
\hline \multicolumn{6}{|c|}{ Smoking/tobacco chewers } \\
\hline Yes $(n=89)$ & 57 & 64 & \multirow[b]{2}{*}{-} & $4.3(0.56-5.0)$ & $<0.0001$ \\
\hline No $(n=1126)$ & 194 & 17.2 & & - & - \\
\hline \multicolumn{6}{|l|}{ Booking status } \\
\hline Unbooked $(\mathrm{n}=837)$ & 175 & 20.9 & \multirow[b]{2}{*}{-} & $1.05(0.77-1.42)$ & $0.7(\mathrm{NS})$ \\
\hline Booked $(n=378)$ & 76 & 20.761 & & - & - \\
\hline \multicolumn{6}{|l|}{ Residence } \\
\hline Rural (n=695) & 184 & 26.5 & \multirow{2}{*}{-} & $1.56(1.12-5.68)$ & 0.01 \\
\hline Urban $(n=520)$ & 67 & 13 & & & - \\
\hline \multicolumn{6}{|l|}{ SES } \\
\hline Class I $(n=20)$ & 0 & 0 & \multirow{5}{*}{-} & & - \\
\hline Class II $(n=30)$ & 3 & 3.3 & & & 0.65 \\
\hline Class III $(\mathrm{n}=680)$ & 13 & 2.2 & & & 0.95 \\
\hline Class IV $(\mathrm{n}=364)$ & 145 & 39.8 & & & 0.02 \\
\hline Class V $(n=121)$ & 90 & 74.38 & & & 0.001 \\
\hline
\end{tabular}


Table 2: Risk factor associated with post-caesarean SSI.

\begin{tabular}{|c|c|c|c|c|c|c|c|c|}
\hline Variables & & $\begin{array}{l}\text { Total } \\
\text { no. of } \\
\text { cases }\end{array}$ & $\begin{array}{l}\text { Total } \\
\text { no. Of } \\
\text { SSI }\end{array}$ & $\begin{array}{l}\text { Incidence } \\
(\%)\end{array}$ & P value & $\begin{array}{l}\text { Superficial } \\
\text { SSI, }(n=154)\end{array}$ & $\begin{array}{l}\text { Deep } \\
\text { SSI, } \\
(n=75)\end{array}$ & $\begin{array}{l}\text { Organ/ } \\
\text { space } \\
\text { SSI, } \\
(\mathrm{n}=22)\end{array}$ \\
\hline \multirow{5}{*}{ BMI $\left(\mathbf{k g} / \mathbf{m}^{2}\right)$} & $<18.5$ & 39 & 0 & 0 & - & & & \\
\hline & $18.5-24.9$ & 364 & 27 & 7.4 & 0.19 & 9 & 18 & 0 \\
\hline & $25.0-29.9$ & 327 & 67 & 20.4 & 0.03 & 50 & 13 & 4 \\
\hline & $30.0-34.9$ & 356 & 104 & 29.2 & 0.01 & 64 & 32 & 8 \\
\hline & $35.0-39.9$ & 134 & 53 & 39.5 & 0.005 & 31 & 12 & 10 \\
\hline \multirow{3}{*}{$\begin{array}{l}\text { Grade of } \\
\text { anemia }\end{array}$} & Mild (8-11) & 764 & 137 & 17.9 & - & 114 & 22 & 1 \\
\hline & $\begin{array}{l}\text { Moderate } \\
(6-8)\end{array}$ & 331 & 79 & 23.8 & 0.02 & 40 & 33 & 6 \\
\hline & Severe $(<6)$ & 117 & 35 & 30 & 0.0027 & 0 & 20 & 15 \\
\hline \multirow{4}{*}{$\begin{array}{l}\text { Type of } \\
\text { procedure } \\
\text { Membrane } \\
\text { rupture } \\
\text { status }\end{array}$} & Routine & 327 & 31 & 9.5 & - & 26 & 5 & 0 \\
\hline & Emergency & 888 & 220 & 24.8 & $<0.0001$ & 128 & 70 & 22 \\
\hline & Yes & 403 & 126 & 31.2 & $<0.0001$ & 46 & 63 & 17 \\
\hline & No & 812 & 125 & 15.3 & - & 108 & 12 & 5 \\
\hline \multirow{3}{*}{$\begin{array}{l}\text { Duration of } \\
\text { membrane } \\
\text { rupture } \\
\text { (Hours) }\end{array}$} & $<12$ & 264 & 26 & 9.8 & 0.02 & & & \\
\hline & $12-24$ & 119 & 80 & 67.2 & $<0.0001$ & & & \\
\hline & $>24$ & 20 & 20 & 100 & $<0.0001$ & & & \\
\hline \multirow{3}{*}{$\begin{array}{l}\text { Grade of } \\
\text { Surgeon }\end{array}$} & $\begin{array}{l}\text { Senior } \\
\text { resident }\end{array}$ & 751 & 156 & 20.7 & $<0.0001$ & 100 & 44 & 12 \\
\hline & Junior resident & 164 & 53 & 32.3 & $<0.0001$ & 12 & 31 & 10 \\
\hline & Consultant & 300 & 42 & 14 & - & 42 & 0 & 0 \\
\hline \multirow{2}{*}{$\begin{array}{l}\text { Intra-op } \\
\text { blood loss } \\
\text { (Mean Hb } \\
\text { difference) } \\
\text { (gm/dl) }\end{array}$} & $>2.2$ & 642 & 184 & 73.3 & $<0.0001$ & 104 & 61 & 17 \\
\hline & $<2.2$ & 573 & 67 & 26.7 & - & 50 & 14 & 5 \\
\hline \multirow{2}{*}{$\begin{array}{l}\text { Blood } \\
\text { transfusion }\end{array}$} & Yes & 537 & 129 & 24 & 0.01 & 101 & 10 & 18 \\
\hline & No & 678 & 122 & 18 & & 53 & 65 & 4 \\
\hline \multirow{2}{*}{$\begin{array}{l}\text { Antibiotic } \\
\text { prophylaxis }\end{array}$} & Yes & 1042 & 177 & 16.9 & $<0.0001$ & 132 & 36 & 9 \\
\hline & No & 173 & 74 & 43 & - & 22 & 39 & 13 \\
\hline \multirow{4}{*}{$\begin{array}{l}\text { Previous } \\
\text { section } \\
\text { Duration of } \\
\text { surgery } \\
\text { (Hour) }\end{array}$} & 1 & 480 & 90 & 18.7 & $0.2(\mathrm{NS})$ & 84 & 6 & 0 \\
\hline & 2 & 68 & 8 & 11.7 & 0.1 (NS) & 6 & 2 & 0 \\
\hline & $<1(\mathrm{n}=700)$ & 700 & 33 & 4.7 & - & 17 & 14 & 2 \\
\hline & $>1(n=515)$ & 515 & 218 & 42.3 & $<0.0001$ & 137 & 61 & 20 \\
\hline \multirow{3}{*}{ Skin incision } & Transverse & 1051 & 192 & 18.2 & - & 133 & 49 & 10 \\
\hline & Vertical & 164 & 59 & 35.9 & $<0.0001$ & 21 & 26 & 12 \\
\hline & Extension & 135 & 28 & 20.7 & 0.00009 & 4 & 16 & 8 \\
\hline \multirow{3}{*}{ Wound class } & Clean & 0 & 0 & 0 & - & 0 & 0 & 0 \\
\hline & $\begin{array}{l}\text { Clean } \\
\text { contaminated }\end{array}$ & 762 & 103 & 13.5 & - & 83 & 18 & 2 \\
\hline & Contaminated & 362 & 98 & 27 & $<0.0001$ & 62 & 29 & 7 \\
\hline
\end{tabular}

Table 3: Distribution of cases according to microorganism in culture.

\begin{tabular}{|lllllll|}
\hline Micro-organism & $\begin{array}{l}\text { Cases in superficial } \\
\text { SSI, }(\mathbf{n}=\mathbf{1 5 4})\end{array}$ & $\%$ & $\begin{array}{l}\text { Cases in deep } \\
\text { SSI, }(\mathbf{n}=\mathbf{7 5})\end{array}$ & $\begin{array}{l}\text { Cases in organ/space } \\
\text { SSI, }(\mathbf{n = 2 2})\end{array}$ & $\%$ \\
\hline Gram positive & 102 & 66.2 & 34 & 45.2 & 12 & 54.2 \\
\hline S. aureus & 49 & 32.4 & 17 & 22.6 & 4 & 18.1 \\
\hline MRSA & 17 & 11.6 & 9 & 12 & - & - \\
\hline Polymicrobial & 22 & 15 & 6 & 8 & 6 & 27.2 \\
\hline Streptococcus & 14 & 9.7 & 2 & 2.6 & 2 & 9 \\
\hline
\end{tabular}

Continued. 


\begin{tabular}{|lllllll|}
\hline Micro-organism & $\begin{array}{l}\text { Cases in superficial } \\
\text { SSI, }(\mathbf{n = 1 5 4})\end{array}$ & $\%$ & $\begin{array}{l}\text { Cases in deep } \\
\text { SSI, (n=75) }\end{array}$ & $\begin{array}{l}\text { Cases in organ/space } \\
\text { SSI, (n=22) }\end{array}$ & $\%$ \\
\hline Gram negative & 53 & 34.4 & 27 & 36 & 9 & 40.9 \\
\hline Klebsiella & 11 & 7.1 & 10 & 13.3 & - & - \\
\hline E-coli & 10 & 6.5 & 2 & 2.6 & - & - \\
\hline P. aeruginosa & 5 & 3.2 & 2 & 2.6 & - & 31.8 \\
\hline Enterococcus & 10 & 6.5 & 1 & 1.3 & 7 & 9 \\
\hline Acinetobacter & 5 & 3.2 & - & - & 2 & - \\
\hline Not specified & 10 & 6.5 & 6 & 8 & - & - \\
\hline Anaerobic cocci & 2 & 1.3 & 6 & 8 & - & - \\
\hline
\end{tabular}

Table 4: Study of cases according to antibiotic sensitivity profile in culture in different category of SSI.

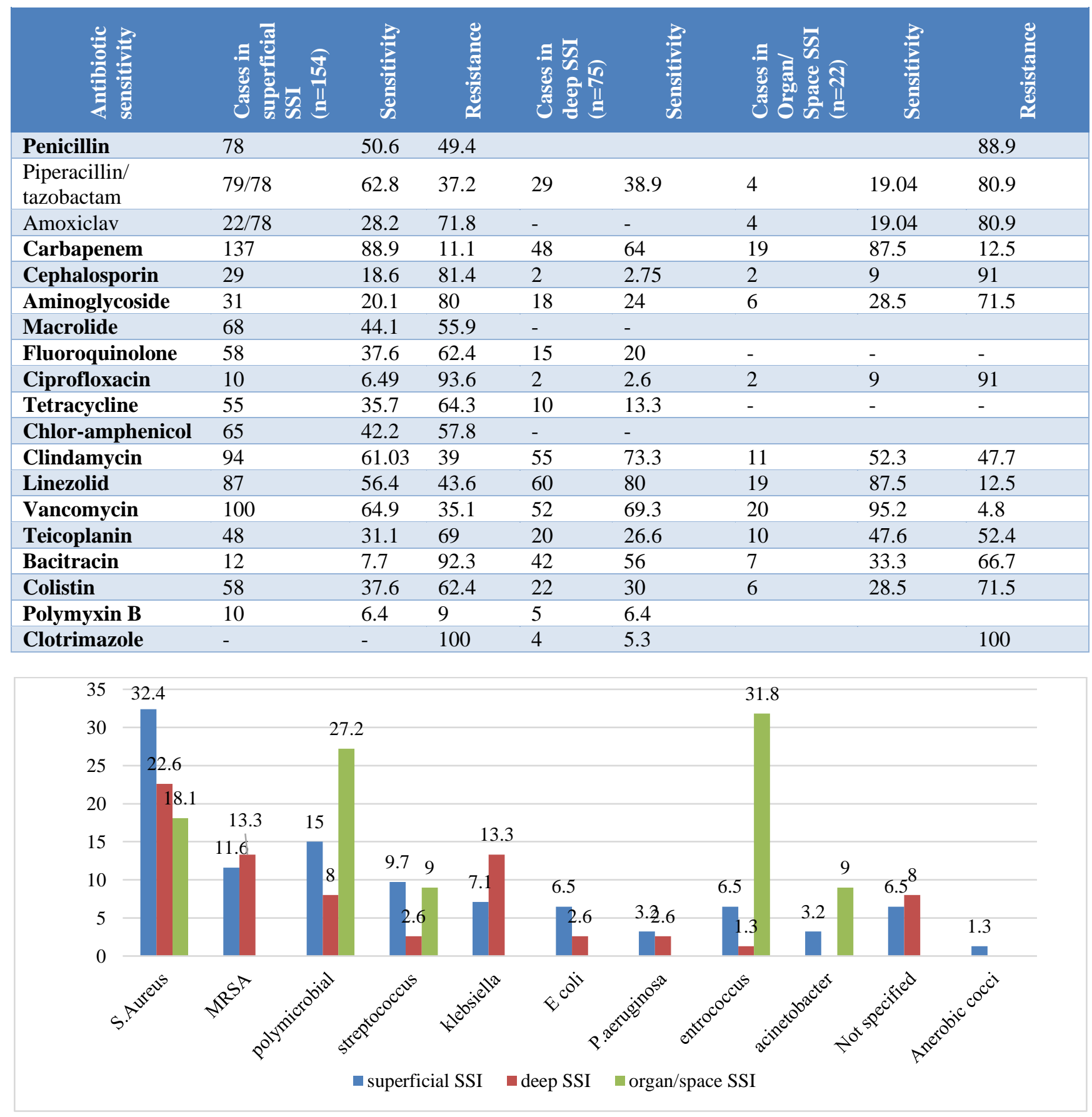

Figure 1: Frequency of pathogenic bacteria isolates from post caesarean SSI. 


\section{DISCUSSION}

During the study period 251 cases developed SSI with incidence of $20.6 \%$. A comparatively low incidence of SSI $(2-13.6 \%)$ has been observed in studies done in developed nations whereas a high incidence in noted in Indian studies by Devjani et al (24.5\%), Shrestha et al (12.6\%), Heetal et al $(28.5 \%)$ the difference in incidence is probably due to differences in population, diversity in indication, hygienic conditions and variation in risk factors associated with SSI. ${ }^{12-14}$

The present study gives an insight of microbiological pattern of post-operative SSI in our hospital and their sensitivity profiles, out of total 251 SSI cases growth in culture was observed in 222 cases $(88.4 \%)$ whereas 29 $(11.5 \%)$ cases showed no growth in culture reason may be the cultures obtained from cases were already receiving antimicrobials as per hospital protocol or wrong technique of sample taking and this could have reduced the pathogens identified. Post caesarean wound infection in this study may not be the true representative of current burden of SSI in our area as most of the cesarean sections are done in CHCs and district hospitals where SSI is likely to be high due to poor adherence to surgical protocol and post-operative management. In demographic profile we did not find any statistically significant difference in incidence of SSI among unbooked $n$ booked cases our results are comparable to Fathia et al, probable reason for this is that intrapartum and postpartum factors are more responsible for developing SSI. ${ }^{1}$ Although good antenatal care decreases the antepartum and intrapartum risks and anemia but for developing SSI in postoperative period prophylactic antibiotics and multiple intrapartum factors are held more responsible.

Among risk factors wound class not only reflects the degree of contamination but also predicts the sterilization status of institute and post-operative morbidity, in our study no cases were taken as clean wound anticipating the contamination of operative wound while entering into genital tract. Maximum cases were clean contaminated wound showing incidence of SSI 13\% while contaminated wounds showed highest incidence of $27 \%$, OR $1.25(1.16-$ $2.56, \mathrm{p}<0.001)$ there is 2.5 fold rise in infection rate when wound class is contaminated, in a study by Amenu et al (2011) 65\% percent of women who developed SSIs had clean contaminated wounds at the time of surgery and the $35.2 \%$ had contaminated or dirty wounds with postcesarean SSI in only $11.4 \%$ cases which is low because they have taken wounds for categorizing wound class from all obstetrics and gynecological surgeries. ${ }^{17}$ Wound class at the time of surgery had a strong statistical association with the severity of SSIs $(\mathrm{p}<0.0001)$.

Post cesarean SSI in our study is categorized into superficial, deep and organ/space SSI as per CDC criteria for SSI from total no of 251 SSI cases incidence of superficial SSI $(\mathrm{n}=154)$ was $61.3 \%$ while for deep SSI $(n=75) 29.8 \%$ and $8.7 \%(n=22)$ organ/space SSI. On analyzing microbiological pattern according to category of SSI we observed, Gram positive organism was highest isolated from superficial SSI $66.2 \%$ while from deep SSI and organ /space SSI gram positive organism isolated was $45.2 \%$ and $54.2 \%$ respectively, gram negative organisms were maximum isolated from organ/space SSI 40.9\% followed by deep and superficial SSI $36 \%$ and $34.4 \%$ respectively.

Incidence of superficial SSI is $61.3 \%$ of all SSI, predominant organism isolated from superficial wounds was Staphylococcus $32.4 \%$ (49/154) cases which was maximum as against other organisms while $11.6 \%$ (17/154) were MRSA. Polymicrobial infection were found in $15 \%$ cases. In gram negative organism; Klebsiella was the most common pathogenic organism it contributed $7.1 \%(11 / 154)$ to superficial SSI while in $16.4 \%(25 / 154)$ cases gram negative bacilli was isolated $E$. coli $(6.5 \%)$, Enterococcus (6.5\%), P. aeruginosa (3.2\%) and Acinetobacter (3.2\%) remained the commonest gramnegative culprits whereas in remaining $20 \%$ cases of gramnegative bacillus, microorganism was not specified. In similar to our study Wloch et al has also shown the maximum pathogenic organism isolated from the surgical site infection is S. aureus (40.4\%) followed by anaerobes, Enterococcus (13.3\%) and Streptococcus (7.4\%). ${ }^{17} \mathrm{~A}$ study by Amandi et al at Nigeria also reported wound infections of $38 \%$ has the highest frequency of S. aureus isolates. $^{18}$

On analyzing the antibiotic sensitivity among organism isolated from the superficial wounds, highest sensitivity was observed for beta lactam antibiotics among them carbapenem contributed to have maximum sensitivity of $88.9 \%$ (137/154); while penicillin group showed sensitivity of $50.6 \%$ (78/154)among them maximum sensitivity was shown by: anti-pseudomonal antibiotic; piperacillin and tazobactam $62.8 \%$ (49/78) and combination of clavulanic acid and amoxicillin $28.2 \%$ (22/78) whereas among beta lactams cephalosporins showed least sensitivity $18.6 \%$ (29/154). The vancomycin (64.9\%) and clindamycin $(61.03 \%)$, drug class contributed to have sensitivity second to carbapenem respectively. Macrolides had sensitivity of $44.1 \%$ (68/154) while sensitivity for aminoglycosides was $20.1 \%$ (31/154). Fluoroquinolones were sensitive in $37.6 \%$ (58/154) cases though, $93.6 \%$ of them showed resistance to ciprofloxacin. Among tetracyclines chloramphenicol was the most sensitive drug 65 of 154 (42.2\%) were sensitive. Many of them were sensitive to miscellaneous antibacterial agents like linezolid $(54.4 \%)$, teicoplanin $(31.1 \%)$, bacitracin $(7.7 \%)$, colistin $(37.6 \%)$, polymyxin B $(6.4 \%)$. Among this group highest sensitivity was observed with linezolid.

The sensitivity pattern of most common pathogen $S$. aureus isolated from superficial wound showed highest sensitivity for linezolid and carbapenems as $97.8 \%(45 / 49)$ and $89.7 \%$ (44/49) respectively while vancomycin which is highly effective against gram positive cocci showed a sensitivity of $71.4 \%$ (35/49) as compared to 
fluoroquinolones which showed an overall sensitivity of $61.2 \%$ (30/49). Among fluoroquinolones newer drugs like gatifloxacin, lomefloxacin, sparfloxacin was found to show more sensitivity against $S$. aureus while $44.8 \%$ (22/49) cases had sensitivity with clavulanic acid and amoxicillin and 32\% (16/49) with cloxacillin. The low percentage sensitivity of $S$. aureus observed in the present study against the following drugs: Tetracycline, Chloramphenicol and Cotrimoxazole was in agreement with the reports published by some workers $[19,20]$ from Nigeria and Naik et al in Eritrea. ${ }^{18}$ Clindamycin is a miscellaneous antibacterial agent acts by inhibiting the protein synthesis it is effective against gram positive cocci in present study it has showed a sensitivity of $42.8 \%$ (21/49).

In 17 of 154 (11.6\%) cases methicillin resistant S. aureus isolated from superficial SSI showed $100 \%$ sensitivity for vancomycin, linezolid $(94.1 \%)$, carbapenem $(88.2 \%)$, clindamycin $(70.5 \%)$ respectively. They showed complete resistance to sulphonamides and quinolones.

From total cases of superficial SSI gram negative bacilli showed highest sensitivity with colistin $88 \%$ and meropenem $80 \%$ while for polymyxin B sensitivity was $76 \%$. Maximum resistance was seen for extended spectrum penicillin except when it is combined with beta lactamase inhibitor. For cephalosporins and aminoglycosides they showed resistance of $80 \%$ and $88.5 \%$ respectively.

We observed that cases with polymicrobial growth $14.2 \%$ (22/154) highest rate of sensitivity was observed with carbapenem $22.7 \%(5 / 22)$ followed by $9 \%(2 / 22)$ with clavulanic acid and amoxicillin, sensitivity in similar proportion was observed with piperacillin, colistin, clindamycin and vancomycin i.e., $13.6 \%$ whereas minimum sensitivity was observed with cephalosporins, linezolid and bacitracin each accounting for $4.5 \%$.

Microbiological pattern in deep SSI presented similar to superficial SSI $S$. aureus was isolated in maximum cases $22.6 \%(17 / 75)$ cases while in $12 \%(9 / 75)$ were MRSA. Polymicrobial infection was found in $6(8 \%)$ cases, from gram negative bacteria's Klebsiella contributed highest to deep SSI $13.3 \%(10 / 75)$ while in $2.6 \%(2 / 75)$ cases $E$. coli was isolated. Gram negative bacteria was responsible for more of deep SSI compared to superficial SSI (25.3\% vs $16 \%)$.

On analyzing the antibiotic sensitivity among organism isolated from deep SSI. Anti-pseudomonal antibiotic piperacillin and tazobactam showed a sensitivity of $38.9 \%$ (29/75) whereas carbapenem accounted for maximum sensitivity of $64 \%$ (48/75) respectively whereas majority of the cases showed resistance against $2^{\text {nd }}$ and $3^{\text {rd }}$ generation cephalosporins, cotrimoxazole and ciprofloxacin remained to be least sensitive $2.6 \%$ $(2 / 75), 5.3 \%(4 / 75)$ and $2.6 \%(2 / 75)$ respectively. The aminoglycoside drug class contributed to have sensitivity of $24 \%(18 / 75)$ while sensitivity for glycopeptides (vancomycin and teicoplanin) was $69.3 \%$ and 26.6 respectively. Many of them were sensitive to miscellaneous antibacterial agents like bacitracin $(56 \%)$, linezolid (80\%), colistin (30\%), polymyxin B $(6.4 \%)$. Among this group highest sensitivity was observed with linezolid $80 \%(60 / 75)$ followed by vancomycin $69.3 \%$ (52/75).

As similar to superficial SSI, the highest sensitivity for $S$. aureus isolated from deep SSI was observed for carbapenems and piperacillin/tazobactam as $88.8 \%$ and $83.3 \%$ respectively, while among clindamycin and linezolid similar proportion of cases were sensitive i.e., $55.55 \%$. Overall minimum sensitivity was seen for clotrimazole $(11.1 \%)$, amoxicillin $(5.5 \%)$ and third generation cephalosporins $(11.1 \%)$ respectively.

$13.3 \%$ (10/75) cases were MRSA showing maximum sensitivity for carbapenem (10/10)100\%, linezolid $(10 / 10) 100 \%$, clindamycin $(8 / 10) 80 \%$, vancomycin $(6 / 10) 60 \%$, teicoplanin (4/10)40\% whereas resistance was seen maximum with quinolones ciprofloxacin (80\%), ceftriaxone (50\%) and clotrimazole (100\%). 36\% (27/75) of gram-negative bacilli isolated from deep SSI showed similar spectrum of antibiotic sensitivity as in superficial SSI highest sensitivity was observed with carbapenem and bacitracin i.e., $100 \%$ while $83.3 \%$ for colistin and while $76 \%$ for polymyxin B. Amikacin which has a broadspectrum antibiotic activity. It is useful for treatment of nosocomial gram-negative infections in our study it was sensitive in $66.6 \%$ cases of deep SSI.

A total $13.3 \%(10 / 75)$ cases isolated Klebsiella species from there wound $70 \%(7 / 10)$ cases among them were resistant to all antibiotics in culture sensitivity report while in $30 \%$ cases it was sensitive to meropenem and linezolid; gentamycin and miscellaneous drug including colistin and polymyxin B showed low sensitivity of $20 \%$. Few cultures reported E. coli and Enterococcus, E. coli species were $100 \%$ sensitive to tetracycline and chloramphenicol while least sensitive to norfloxacin, gentamycin and amikacin. Enterococcus species was isolated in only one sample of deep SSI, they were highly resistant to penicillin (100\%), ciprofloxacin (100\%) and aminoglycosides (85.2\%) and showed sensitivity for nitrofurantoin, vancomycin, clindamycin and teicoplanin. E. coli and gram-negative bacteria are commensal of the normal vaginal flora. Our study shows that $E$. coli has not only developed resistance to penicillin but also to third generation cephalosporins. This could be because of prolonged administration of cephalosporins which had led to emergence of resistant strains of E. coli.

Anaerobic cocci most clinical isolates are identified to species in the genus Pepto streptococcus. They are part of the normal flora of all mucocutaneous surfaces and are often isolated from infections such as deep organ abscesses, obstetric and gynecological sepsis. They accounted for $8 \%$ of total SSI cases among these cases 
$50 \%$ were resistant to all antibiotics while the remaining were highly sensitive to chloramphenicol (50\%), teicoplanin, carbapenem, gentamycin and fluoroquinolones each accounting for $33.3 \%$ sensitivity respectively.

Among pathogenic organism isolated from organ/space SSI gram negative organisms contributed maximum, Enterococcus was the most common $31.8 \% \quad(7 / 22)$ followed by Polymicrobial infection isolated in 6 (27.2\%) cases while among gram positive cocci $S$. aureus contributed to $18.1 \%(4 / 22)$ cases and in $9 \%(2 / 22)$ streptococcus species were isolated. A few cultures yielded the growth of Acinetobacter (9\%) which is a pleomorphic aerobic gram-negative bacillus commonly isolated from the hospital environment and hospitalized patients.

Glycopeptides (vancomycin) showed the highest sensitivity among cases of organ/space SSI accounting for 95.2\% (20/21). Among these cases carbapenems and linezolid showed equal sensitivity of $87.5 \%$ which is extremely high when compared to beta lactam antibiotics including anti-pseudomonal antibiotic; piperacillin and tazobactam (19.04\%) and ampicillin/sulbactam (33.3\%). In aminoglycoside drug class sensitivity to amikacin was $28.5 \%(6 / 21)$ which is comparable in all 3 classes of SSI while sensitivity for miscellaneous drugs like bacitracin $(33.3 \%)$, clindamycin $(52.3 \%)$ and colistin $(28.5 \%)$ was acceptable.

Most common organism isolated from organ/space SSI was Enterococcus they are gram positive Diplococcus. They are commensal organism of intestine. They have a high level of intrinsic antibiotic resistance. Some enterococci are intrinsically resistant to $\beta$-lactam based antibiotics (penicillin's, cephalosporins, carbapenems), as well as many aminoglycosides. In our study highest sensitivity for Enterococcus among cases of organ/space SSI was observed for carbapenem $100 \%$, vancomycin as $100 \%(7 / 7)$ and teicoplanin $85.7 \%$ (6/7) respectively whereas all the species of Enterococcus showed resistance to beta lactam antibiotics penicillin (88.9\%), cephalosporin group (78.6\%), ciprofloxacin (90\%). They also showed sensitivity for bacitracin, colistin, polymyxin $\mathrm{B}$, and neomycin as $42.8 \%, 42.8 \%, 71.4 \%$, and $57.1 \%$ respectively.

In $6 / 22(27.2 \%)$ cases polymicrobial growth was isolated it showed maximum sensitivity for linezolid (100\%), carbapenem $(83.3 \%)$, amikacin $(83.3 \%)$, vancomycin $(83.3 \%)$ and clindamycin $66.6 \%$.

Staphylococcus was isolated from $18.1 \%$ (4/22) cases having maximum sensitivity for carbapenem $87.5 \%$ and linezolid $87.5 \%$ while for cephalosporins and aminoglycoside it has developed resistance in $91 \%$ and $71.5 \%$ of cases respectively. Few cultures have reported Acinetobacter species from organ/space SSI which is a common bacterium for nosocomial infection in our study it has been isolated from 2 cases of organ SSI and has shown resistance for all antibiotics. In our study the microbial etiology of post CS SSIs has been shown to be diverse, being associated with both vaginal microorganisms such as E-coli, group B Streptococcus (GBS) and Enterococcus spp, or with nasopharyngeal flora such as $S$. aureus or skin flora such Staphylococcus epidermidis, $S$. aureus has been found to be the most common cause of SSI post CS. Other organisms such as Klebsiella spp, Pseudomonas aeruginosa, and Enterococcus spp, show a variable distribution pattern. Isolation of bacteria that are not present in the genital tract such as pseudomonas, which are found in the hospital environment and MRSA and Acinetobacter found in the skin shows the iatrogenic etiology of wound infection these can be reduced by decreasing the duration of hospital stay and by practicing more strict aseptic measures.

In present study most common micro-organism isolated was gram positive $S$. aureus isolated among superficial and deep SSI while among gram negative organism's Enterococcus was the most common organism isolated from organ space SSI. In contrast to Indian study by Devajani et al gram-negative bacilli were the commonest finding. ${ }^{14}$ The commonest isolate was Acinetobacter species $(32.03 \%)$ followed by $S$. aureus and coagulase negative Staphylococcus $(21.09 \%)$. In our study S. aureus has shown maximum sensitivity for carbapenems, linezolid and vancomycin these findings are in contrast with a Nigerian study Olufunmiola et al, which reported that $S$. aureus isolates were highly sensitive to cephalosporins and amoxicillin. Another study by Akinkunmi et al observed that $S$. aureus isolates are highly sensitive to fluoroquinolones and cephalosporins. ${ }^{22,23}$

Among gram negative bacteria in present study Klebsiella and Enterococcus was most common isolates showed maximum sensitivity for carbapenems, colistin and vancomycin and were highly resistant to ciprofloxacin, aminoglycosides, co-trimoxazole and to third generation cephalosporins. However, in study by Olufunmiola et al observed that gram-negative isolates were overly sensitive to cephalosporins and fluoroquinolones. ${ }^{22}$ This disparity in resistant pattern observed in this study may be due to injudicious use of first line antimicrobial drugs. High sensitivity to imipenem, linezolid and vancomycin may be due to the limited exposure of these drugs.

Our study has thoroughly observed the microbiological and antibiotic sensitivity pattern of all post cesarean SSI distinctly in each category i.e., superficial, deep and organ space SSI as per CDC criteria in our institute providing a more elaborated and detailed spectrum of microbiological and antibiotic sensitivity pattern. However, bacterial resistance mechanisms may exist and contribute to evade the effect of prophylactically administered antibiotics. Therefore, it becomes imperative to understand the local antibiotic susceptibility patterns existing in the community so as to design a suitable local antibiotic policy to decrease the frequency of surgical site infection. 
Limitations of the study needs more multicentric studies in our area for generalization of results.

\section{CONCLUSION}

In conclusion, our tertiary centre had post caesarean SSI rate of $20.6 \%$ which was high. Among gram positive $S$. aureus, MRSA and in gram negative Klebsiella pneumonia and Enterococcus were the commonest isolates from post-caesarean wound infection. Proper antenatal care is needed to reduce intrapartum and postpartum risk factors for developing SSI. Antibiotic prophylaxis and postoperative antibiotic protocol policy should be individualised to institutions as per there microbiological and sensitivity pattern, hospitals should have their SOPs regarding SSI and infection control policies should be strict enough to prevent unnecessary iatrogenic nosocomial infections. This step will be beneficial to both hospitals and community as it would lead to lower the burden associated with SSI.

Funding: No funding sources

Conflict of interest: None declared

Ethical approval: The study was approved by the Institutional Ethics Committee

\section{REFERENCES}

1. Mangram AJ, Horan TC, Pearson ML, Silver LC, Jarvis WR. Guideline for Prevention of Surgical Site Infection, 1999. Centers for Disease Control and Prevention (CDC) Hospital Infection Control Practices Advisory Committee. Am J Infect Control. 1999;27(2):97-132.

2. NICE guidelines [CG132]. Caesarean Section. Clinical Guideline. London: RCOG. 2011.

3. Srun S, Sinath Y, Seng AT. Surveillance of postcaesarean surgical site infections in a hospital with limited resources, Cambodia. J Infect Dev Ctries. 2013;7:579-85.

4. National Nosocomial Infections Surveillance (NNIS) System Report, data summary from January 1992 through. 2004;2004

5. Ganguly PS, Khan Y, Malik A. Nosocomial infection and hospital procedures. Indian $\mathbf{J}$ Common Med. 2000;990-1014.

6. Ramasubramanian V, Iyer V, Sewlikar S, Desai A. Epidemiology of healthcare acquired infection-An Indian perspective on surgical site infection and catheter related blood stream infection. Indian J Basic App Med Res. 2014;3(4):46-63.

7. Petter EC, Farret TCF, Scherer JS, Antonello VS. Fatores relacionados a infecções de sítio cirúrgico após procedimentos obstétricos. Scientia Medica (Porto Alegre). 2013;23:28-33.

8. Baaqeel H, Baaqeel R. Timing of administration of prophylactic antibiotics for caesarean section: a systematic review and meta-analysis. BJOG. 2013;120;661-9.
9. Ako-Nai AK, Adejuyigbe O, Adewumi TO, Lawal OO. Sources of intra-operative bacterial colonization of clean surgical wounds and subsequent post-operative wound infection in a Nigerian hospital. East Afr Med J. 1992;69:500-7.

10. Egah DZ, Bello CS, Banwat EB, Allanana JA. Antimicrobial sensitivity pattern for Staphylococcus Aureus in Jos Nigeria. Niger J Med. 1999;8:58-61.

11. Ogunsola FT, Oduyebo O, Iregbu KC, Coker AO, Adetunji A. A review of nosocomial infection at the Lagos university teaching hospital: Problems and strategies for improvement. J Nig Infect Contr Assoc. 1998;1:14-20.

12. Shrestha S, Shrestha R, Shrestha B, Dongol A. Incidence and Risk Factors of Surgical Site Infection Following Cesarean Section at Dhulikhel Hospital. 2014;12(2):1136.

13. Heethal J, Sarala N, Kumar TN, Hemalatha M. Pattern of antimicrobial use in caesarean section in a tertiary care hospital in rural south India. Int $\mathbf{J}$ of Biomed Res. 2010;2:57-61.

14. Devjani De, Saxena S, Mehta G, Yadav R, Dutta R. Risk Factor Analysis and Microbial Etiology of Surgical Site Infections following Lower Segment Caesarean Section. Int J Antibiotics. 2013;283025:6.

15. Fathia E Al. Jama Risk factors for wound infection after lower segment cesarean section. Qatar Med J. 2012;2012(2):26-31.

16. Amenu D, Belachew T, Araya F. Surgical site infection rate and risk factors among obstetric cases of Jimma University specialized hospital, southwest Ethiopia. Ethiop J Health Sci. 2011;21:91-100.

17. Wloch C, Wilson J, Lamagni T, Harrington P, Charlett A, Sheridan E. Risk factors for surgical site infection following caesarean section in England: Results from a multicentre cohort study. BJOG. 2012;119:1324-33.

18. Amadi ES, Ikeagwu IJ, Iroha IR. Antibiotic sensitivity pattern of Staphylococcus aureus in Abakiliki, Nigeria. Pak J Med Sci. 2008;24(2):231-5.

19. Obiazi HAK, Nmorsi OPG, Ekundayo AO, Ukwuandu NCD. Prevalence and antibiotic susceptibility pattern of Staphylococcus aureus from clinical isolates grown at 37 and $44 \mathrm{oC}$ from Irrua, Nigeria. Afr $\mathrm{J}$ Microbiol Res. 2007:57-60.

20. Ndip RN, Ebah LME, Onile BA. Antibiogram of Staphylococcus aureus from clinical Syndromes in Ilorin, Nigeria. J Med Lab Sci. 1997;6:24-6.

21. Naik D, Teclu A. A study on antimicrobial susceptibility pattern in clinical isolates of Staphylococcus aureus in Eritrea. Pan Afr Med J. 2009;3(1).

22. Olufunmiola BM, Olugbenga AO, Adeyankinnu AF. Bacteria agents of surgical site infection in south-west Nigeria. Am J Biomed Sci. 2013;5(4):217-25.

23. Akinkunmi EO, Adesunkanmi AR, Lamikanra A. Pattern of pathogens from surgical wound infections in a Nigeria hospital and their antimicrobial susceptibility profiles. Afr Health Sci. 2014;14(4):802-9.

Cite this article as: Thakur N, Kujur A.

Microbiological and antibiotic sensitivity pattern of surgical site infection following caesarean section in a tertiary care center of Chhattisgarh. Int J Reprod Contracept Obstet Gynecol 2021;10:2638-46. 